\title{
Crowding-Out dos Trabalhadores com Baixa Qualificação no Brasil*
}

\section{Crowding-Out of Low-Skilled Workers in Brazil}

\author{
Maria Cristina Cacciamali** \\ Tânia de Toledo Lima*** \\ Fábio Tatei****
}

\begin{abstract}
Resumo: Este estudo busca verificar se ocorreu o efeito crowding-out nas regiões metropolitanas do Brasil nas recessões de 1998, 2002 e 2009, sob a abordagem de Schult (2005). Os resultados demonstram que cada recessão apresentou peculiaridades quanto ao efeito na probabilidade de transição dos indivíduos desocupados para a condição de ocupados. Contudo, o efeito crowding-out ocorreu em 2009. Vale ressaltar que esse efeito ocorreu mesmo sob um cenário de recessão com elevação do prêmio por qualificação.
\end{abstract}

Palavras-chave: Efeito crowding-out. Prêmio por qualificação. Probabilidade de transição.

\begin{abstract}
This study aims to verify if the crowding-out effect occurred in Brazilian's metropolitan regions Brazil during recessions in 1998, 2002 and 2009, under the approach Schult (2005). The results demonstrate that each recession presented peculiarities in the probability of transition from unemployment to employment for individuals. However, the crowding-out effect occurred in 2009. It is also worth to mention that this effect occurred even under a scenario of recession with rising premium for qualification.
\end{abstract}

Keywords: Crowding-out effect. Premium for qualification. Probability of transition.

JEL Classification: C14; J64.

\footnotetext{
* Pesquisa realizada sob os auspícios do CNPq.

** Doutora em Economia pela Universidade de São Paulo (USP). Livre docente da USP. Coordenadora do Núcleo de Estudo e Pesquisa de Política Internacional, Estudos Internacionais e Políticas Comparadas (Nespi/USP). Bolsista sênior CNPq. E-mail: cciamali@uol.com.br

*** Mestra em Economia pela Universidade Federal de São Carlos (UFSCar). Membro do Núcleo de Estudo e Pesquisa de Política Internacional, Estudos Internacionais e Políticas Comparadas (Nespi/ USP). E-mail: taniatl@al.insper.edu.br

**** Mestre em Integração da América Latina pela Universidade de São Paulo (USP). Pesquisador pleno do Núcleo de Estudo e Pesquisa de Política Internacional, Estudos Internacionais e Políticas Comparadas (Nespi/USP). E-mail: ftatei@usp.br
} 


\section{1 lntrodução}

O objetivo deste estudo é verificar se o efeito crowding-out dos trabalhadores com baixa qualificação ocorreu no Brasil durante os períodos de recessão econômica de 1998, 2002 e 2009. ${ }^{1}$ Para tanto, é testada uma hipótese para esses anos com base na teoria de ciclos de negócios: a mudança na probabilidade de transição dos desocupados para a situação de ocupados, tanto para trabalhadores qualificados, quanto para os menos qualificados. Ressalva-se que a qualificação dos trabalhadores é uma variável complexa e de difícil mensuração com as bases de dados disponíveis no Brasil, no entanto diversos estudos apontam que, guardada as devidas proporções, o grau de escolaridade pode ser usada como proxy para a qualificação dos trabalhadores (FERNANDES; MENEZES FILHO, 2012; REIS, 2006a).

Posto isso, a análise da probabilidade de transição levou em consideração o estreitamento do diferencial de salários entre trabalhadores qualificados e menos qualificados no período. Essa escolha teve a ver com o efeito do aumento da oferta de trabalhadores qualificados sobre a probabilidade do desocupado se inserir no mercado de trabalho. A premissa foi testada por meio do modelo parametric hazard regression, conforme especificado por Blossfeld e Rohwer (2002), para estimar a situação da desocupação para a ocupação, e sob a abordagem de Schult (2005). ${ }^{2}$ As estimativas foram realizadas para os anos de 1998, 2002 e 2009, em seis regiões metropolitanas (RM) brasileiras, a partir dos microdados da Pesquisa Mensal de Emprego (PME), levantamento realizado pelo Instituto Brasileiro de Geografia e Estatística (IBGE).

A principal relevância do estudo está em verificar a transição dos desocupados para ocupados para os grupos qualificados e menos qualificados, uma vez que se busca verificar se há distinção entre a transição nos períodos de recessão e se os fatores que influenciam essa transição são os mesmos, independentemente do aumento da oferta de trabalho e das distinções entre as recessões brasileiras destacadas. A importância em se conhecer tal fato pauta-se em dois aspectos: no primeiro, verifica-se se características individuais são relevantes, independente das características da recessão; no segundo, identifica-se se as características referentes à estrutura do mercado de trabalho apresentam algum papel nessa transição diante do cenário de recessão. No primeiro caso, busca-se identificar algum grupo com características comuns que podem ser foco para políticas públicas. No segun-

1 A crise internacional que se concretiza com a quebra do Lehman-Brothers ocorre em 2008, contudo o crescimento do PIB brasileiro foi afetado apenas em 2009.

2 Segundo Schult (2005), é possível analisar a mudança na probabilidade de transição dos desocupados para os ocupados e vice-versa, mas no caso brasileiro a abordagem desse modelo não é adequada por ausência de variáveis relevantes na base de dados, tal como número de funcionários na empresa onde o funcionário trabalha. 
do caso, busca-se verificar se a qualificação apresenta relevância diante de um contexto de recessão, ou se pode ocorrer algum prejuízo ao trabalhador, no que se refere às habilidades, num cenário de recessão, com ou sem deterioração do mercado de trabalho.

O presente artigo encontra-se estruturado, além desta introdução, em mais quatro seções: a segunda apresenta interpretações do que ocasiona o aumento da taxa de desocupação dos menos qualificados; a terceira descreve a amostra e o modelo parametric hazard regression aplicado para estimar a transição da situação da desocupação para a ocupação; a quarta analisa os resultados da hipótese de crowding-out no mercado de trabalho brasileiro em períodos de crise econômica; e, por fim, a quinta traz as considerações finais.

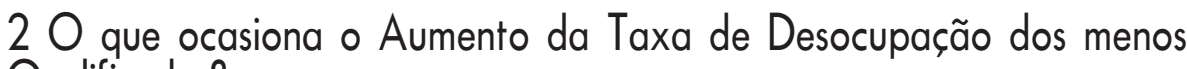 Qualificados?}

O aumento da taxa de desocupação dos trabalhadores menos qualificados nos períodos de recessão econômica é explicado frequentemente por duas interpretações. Berman, Bound e Griliches (1994), Nickel (1986) e Nickel e Bell (1995) argumentam que os empregadores em períodos de recessão preferem perder trabalhadores de menor qualificação àqueles de qualificação mais elevada. A razão disso está nos custos de demissão (associados aos salários), recontratação e treinamento sempre maiores para trabalhadores de maior qualificação e custos quase fixos de mão de obra mais elevados, segundo a definição de Oi (1962). Assim, ao longo de uma recessão, os trabalhadores menos qualificados seriam os primeiros a serem demitidos, o que provocaria o aumento relativo da taxa de desocupação desse grupo frente aos demais, pois não há demanda para esse tipo de trabalhador.

A segunda interpretação proporciona outra racionalização. Não é incomum que trabalhadores com maiores qualificações ocupem postos de trabalho que requerem menores qualificações devido à oferta restrita de postos de trabalho qualificado. Nessa situação, as oportunidades para trabalhadores de baixa qualificação diminuem mesmo quando ocorre aumento da oferta de postos de trabalho menos qualificados. Essa abordagem é denominada de hipótese crowding-out, que tem por base o modelo de competição por postos de trabalho (teoria da fila) de Thurow (1975). Entretanto, vale ressaltar que o crowding-out não está restrito ao processo de contratação, podendo estar também ligado ao processo de demissão. No primeiro caso, o excesso de mão de obra qualificada leva os empregadores a contratar esse tipo de trabalhador mesmo para vagas que seriam preenchidas por trabalhadores menos qualificados. E no segundo caso - processo de demissão -, os empregadores demitem os trabalhadores de baixa qualificação no intuito de elevar a estrutura de qualificação do seu quadro de funcionários. A consequência 
é a redução das oportunidades de emprego para trabalhadores de menor grau de qualificação nas duas situações.

Alterações no prêmio pela qualificação, logo, nos diferenciais entre os salários dos trabalhadores qualificados e menos qualificados, estão relacionadas ao perfil de oferta e demanda no mercado de trabalho (CARD; LEMIEUS, 2001; KATZ; MURPLY, 1992; MANACORDA; SÁNCHEZ PÁRAMO; SCHADY, 2010), que exercem influência sobre os desocupados retardando ou reduzindo seu tempo de desocupação. Os diferenciais de salário médio tendem a se estreitar ao longo do tempo, seja porque o ritmo de criação de postos de trabalho mais qualificado é menor do que a expansão de trabalhadores qualificados egressos do sistema educacional, seja porque, como no caso do mercado de trabalho brasileiro, houve aumento na oferta de trabalho qualificado estreitando, assim, o "prêmio pela qualificação", sobretudo no período entre 1990 e 2000.

Destacam-se adicionalmente dois conjuntos de fatores que podem afetar a estrutura de trabalho no Brasil. Primeiramente, devem-se considerar os efeitos do progresso técnico sobre a demanda por qualificação. Vários estudos retratam a complementaridade entre progresso técnico e demanda por profissionais qualificados. Entre inúmeros autores que demonstram essa relação, destacam-se Autor, Levy e Murnane (2003), para os Estados Unidos, e Menezes Filho e Rodrigues Júnior (2003) para o Brasil. Rodrigues, Oliveira e Albuquerque (2008), além disso, mostram elevação do bônus dos profissionais em ocupações intensivas em tecnologia, enquanto Giovanetti e Menezes Filho (2007) apontam que a liberalização comercial tornou os setores de máquinas e insumos mais intensivos em tecnologia, o que implicou aumento na ocupação de trabalhadores mais qualificados.

O segundo conjunto de fatores que atua sobre a estrutura de trabalho relaciona-se com as instituições. Essas afetam a oferta e a demanda por qualificação no mercado de trabalho por meio das alterações nos custos e preços relativos, como a mudança do salário mínimo, que pode alterar as oportunidades para as mulheres menos qualificadas (DINARDO; FORTIN; LEMIEUX, 1996), ou pelas mudanças em outras regulamentações de mercado laboral, como legislação sindical, trabalhista ou previdenciária, ou, ainda, com intervenções no mercado de bens, como tarifas ou incidência de impostos para um determinado setor econômico.

Mais recentemente, no contexto internacional, Katz e Margo (2013) destacam que, apesar da "polarização" no mercado de trabalho norte-americano desde a década de 1980, a desqualificação não tem ocorrido, uma vez que houve elevação na ocupação independente da qualificação, enquanto novas evidências sugerem que houve um aumento na demanda por trabalhadores qualificados mais rápido que a elevação da oferta de trabalhadores qualificados antes da Primeira Guerra Mundial. Por sua vez, Dolado, Felgueroso e Jimeno (2000) mostram como a combinação de excesso de mão de obra qualificada e relações laborais rígidas 
influenciaram o elevado grau de desocupação dos trabalhadores de menor qualificação na Espanha, sobretudo dos mais jovens, além de aumentar o percentual de trabalhadores qualificados em ocupações consideradas não adequadas para sua capacitação.

Para o caso brasileiro, Reis (2006b) observa, com base nos dados da PNAD de 1990 a 1999, que alterações nas produtividades por grupos de qualificação levaram a um aumento na demanda por trabalhadores qualificados em comparação aos semiqualificados e não qualificados, resultando em redução da desocupação dos qualificados e aumento da demanda desses trabalhadores em relação aos demais grupos. Também utilizando dados da PNAD, Pecora (2012) verifica que a diferença entre os salários dos trabalhadores de elevada qualificação e os salários dos demais trabalhadores aumentou no Brasil entre 1992 e 2001, impulsionada principalmente pelo aumento da demanda dos trabalhadores qualificados. Por outro lado, o aumento da oferta de trabalhadores qualificados acarretou a redução dessa diferença de salários entre 2002 e 2009. Fernandes e Menezes (2012) investigam a alocação de trabalhadores de acordo com a educação e o salário, sendo que os resultados indicam que houve um aumento na demanda por trabalhadores qualificados nas tarefas complexas e elevação da demanda por trabalhadores intermediários em tarefas mais simples. Entretanto, de 1981 a 2009, os autores observaram que, sem os choques, o salário relativo dos trabalhadores intermediários teria se reduzido ainda mais, enquanto o dos trabalhadores qualificados decresceu.

\section{Mudanças na Estrutura de Qualificação e Modelo Empregado}

A amostra do presente estudo foi construída a partir da PME, tendo em consideração as diferenças na metodologia a partir de 2002, ao considerar os anos de 1998, 2002 e 2009 para a análise. Antes de se considerar os dados para análise, a amostra foi tratada sob a abordagem de Ribas e Soares (2008) no intuito de reduzir possíveis atritos na construção do painel. Sendo assim, para o estudo são consideradas as RM de Recife, Salvador, Belo Horizonte, São Paulo, Rio de Janeiro e Porto Alegre. O trabalhador - ocupado ou desocupado - foi classificado segundo o seu grau de qualificação em virtude de seu nível de escolaridade. A categoria de "menos qualificados" compreende os trabalhadores que dispõem de nível de escolaridade equivalente até o ensino médio completo, e a de "qualificados" compreende os trabalhadores com pelo menos o ensino superior incompleto. A classificação foi realizada para cada RM no intuito de compreender a dispersão do grupo na amostra entre elas. Em seguida, foram considerados os possíveis fatores que afetam a mudança na probabilidade de transição de desocupado para ocupado e vice-versa, dado os dois grupos. 
As Tabelas 1 e 2 mostram as mudanças que ocorreram no percentual dos desocupados qualificados e menos qualificados, e na média dos anos de estudo, nos anos de 1998, 2002 e 2009, nas seis RM pesquisadas pela PME. Observa-se, ao comparar 1992 com 2008, que a média dos anos de estudo dos desocupados menos qualificados aumentou no período, enquanto que a média dos anos de estudo dos desocupados qualificados pouco se alterou e a sua proporção relativa no total aumentou. Esses resultados permitem considerar a aplicação da hipótese de crowding-out.

Tabela 1 - Proporção dos indivíduos desocupados: qualificados e com menor qualificação

\begin{tabular}{lccc|ccc}
\hline \multirow{2}{*}{$\begin{array}{l}\text { Regiões } \\
\text { metropolitanas }\end{array}$} & \multicolumn{3}{l|}{ \% de desocupados } & \multicolumn{3}{l}{ \% de desocupados menos } \\
& qualificados & & \multicolumn{2}{l}{ qualificados } & \\
& $\mathbf{1 9 9 2}$ & $\mathbf{1 9 9 8}$ & $\mathbf{2 0 0 9}$ & $\mathbf{1 9 9 2}$ & $\mathbf{1 9 9 8}$ & $\mathbf{2 0 0 9}$ \\
\hline Recife & $3,78 \%$ & $7,18 \%$ & $7,25 \%$ & $96,22 \%$ & $92,82 \%$ & $92,75 \%$ \\
Salvador & $2,71 \%$ & $5,02 \%$ & $12,93 \%$ & $97,29 \%$ & $94,98 \%$ & $87,07 \%$ \\
Belo Horizonte & $4,51 \%$ & $9,63 \%$ & $15,81 \%$ & $95,49 \%$ & $90,37 \%$ & $84,19 \%$ \\
Rio de Janeiro & $8,06 \%$ & $8,82 \%$ & $12,96 \%$ & $91,94 \%$ & $91,18 \%$ & $87,04 \%$ \\
São Paulo & $6,43 \%$ & $7,52 \%$ & $14,61 \%$ & $93,57 \%$ & $92,48 \%$ & $85,39 \%$ \\
Porto Alegre & $5,68 \%$ & $8,33 \%$ & $13,48 \%$ & $94,32 \%$ & $91,67 \%$ & $86,52 \%$ \\
\hline
\end{tabular}

Fonte: Elaboração própria a partir dos microdados da PME (IBGE, 2015b).

Tabela 2 - Média dos anos de estudo dos indivíduos desocupados: qualificados e com menor qualificação

\begin{tabular}{lccc|ccc}
\hline \multirow{2}{*}{$\begin{array}{l}\text { Regiões } \\
\text { metropolitanas }\end{array}$} & \multicolumn{3}{c|}{ Média dos anos de estudo } & \multicolumn{3}{c}{ Média dos anos de estudo } \\
& $\mathbf{1 9 9 2}$ & $\mathbf{1 9 9 8}$ & $\mathbf{2 0 0 9}$ & $\mathbf{1 9 9 2}$ & $\mathbf{1 9 9 8}$ & $\mathbf{2 0 0 9}$ \\
\hline Recife & 14,47 & 14,64 & 13,60 & 6,10 & 6,86 & 9,38 \\
Salvador & 14,14 & 14,53 & 13,78 & 6,57 & 6,64 & 9,09 \\
Belo Horizonte & 13,88 & 13,96 & 14,08 & 6,06 & 7,07 & 9,07 \\
Rio de Janeiro & 14,45 & 13,96 & 14,00 & 6,95 & 7,76 & 9,02 \\
São Paulo & 14,11 & 14,47 & 13,94 & 6,33 & 7,53 & 8,99 \\
Porto Alegre & 13,86 & 13,94 & 14,13 & 6,61 & 7,36 & 8,70 \\
\hline
\end{tabular}

Fonte: Elaboração própria a partir dos microdados da PME (IBGE, 2015b).

A metodologia empregada para se estimar a transição da situação da desocupação para a ocupação, e vice-versa, assenta-se no modelo parametric hazard 
regression, conforme especificado por Blossfeld e Rohwer (2002). ${ }^{3}$ Dessa forma, é necessário inicialmente determinar a taxa de risco que expressa a probabilidade instantânea da ocorrência de um evento num dado intervalo de tempo, condicionado ao fato de dado evento não ter ocorrido anteriormente. Como os dados são provenientes da PME, este estudo utiliza o tempo discreto na estimação da taxa de risco, considerando apenas a primeira e a segunda entrevistas em que cada indivíduo aparece na pesquisa. Assim, tem-se:

$$
h(t)=\lim _{\Delta t \rightarrow 0} \frac{P(t \leq T<t+\Delta t \mid T \geq t)}{\Delta t}
$$

Vale ressaltar que, segundo Biewen e Wilke (2005), a estimação que considera o tempo discreto não considera a heterogeneidade não observada e pode gerar estimadores viesados. Para escapar desse viés, considerou-se a estimação paramétrica da função de risco por meio de uma forma específica. Esse uso permite controlar a correlação entre trabalhadores, pois ela pode ocorrer quando se consideram diferentes períodos de duração da desocupação para um grupo de respondentes. Assim, tomou-se a seguinte forma:

$$
\ln \left(t_{i}\right)=x(t) \beta_{i}+\ln \left(\tau_{i}\right)
$$

em que $\ln \left(t_{i}\right)$ é o logaritmo do tempo gasto na situação original até se mover para a situação $i ; x(t)$ é um vetor linha de variáveis covariadas variando no tempo $t$; e $\ln \left(\tau_{i}\right)$ é um componente de erro. Vale ressaltar que a distribuição do componente de erro é determinada de acordo com o modelo adotado para a estimação da forma funcional. Por exemplo, considerando que a distribuição de $\tau_{i}$ é uma log-normal, ter-se-á que $\ln \left(\tau_{i}\right)$ seguirá uma distribuição normal. $\beta_{i}$ mensura o impacto das covariadas no período de duração na situação original. Logo, a variação de $\beta_{i}$ se caracteriza da seguinte forma: se for igual a zero, as covariadas não apresentam nenhum impacto; se for positivo, as covariadas apresentam impacto positivo no período de duração, esperando-se que o efeito ocorra algum tempo depois; e se for menor que zero, espera-se que o impacto ocorra em breve.

Por fim, consideraram-se as seguintes variáveis dependentes para a estimação da transição da situação de desocupação dos trabalhadores menos qualificados para a situação de ocupados:

3 Em especial, foram considerados os capítulos 3 e 7. O capítulo 3 foi utilizado na compreensão dos métodos não paramétricos, enquanto do capítulo 7 foram considerados os métodos de estimação paramétricos, mais especificamente, log-logística e log-normal, para a análise dos dados de duração do desemprego quando ocorre a transição para o emprego, e vice-versa. 

a) gênero;
b) cor da pele; ${ }^{4}$
c) idade;
d) idade ao quadrado;
e) taxa de desocupação;
f) dummy para indivíduos que apresentavam menor qualificação e se matri- cularam em algum curso de qualificação profissional;
g) interação do nível de qualificação com taxa de desocupação;
h) dummy para a situação anterior do trabalhador, ou seja, se estava empre- gado anteriormente ou não;
i) dummy para trabalhadores que nunca estiveram ocupados anteriormente;
j) interação das dummies para as RM, considerando a de São Paulo como referência, com a variação da atividade industrial de cada uma; ${ }^{5}$
k) dummy para o mês de dezembro; ${ }^{6}$
1) proporção de trabalhadores que estavam em treinamento ou buscando melhor qualificação;
m) prêmio por qualificação do trabalhador, que é dado pelo quociente entre o salário médio do trabalhador com curso superior completo e o salário médio do indivíduo com ensino médio completo;
n) proporção de trabalhadores qualificados em relação aos menos qualifi- cados que estavam desocupados na primeira e na segunda entrevistas e conseguiram trabalho na terceira ou na quarta entrevistas.

\section{Maior Qualificação da Força de Trabalho leva ao Crowding-Out}

O presente estudo se propôs a verificar se o efeito crowding-out dos trabalhadores com baixa qualificação ocorreu no Brasil durante os períodos de recessão econômica de 1998, 2002 e 2009. Logo, com base na teoria de ciclos de negócios, foi estimada a mudança na probabilidade de transição dos desocupados, sejam trabalhadores qualificados e menos qualificados, para a situação de ocupados. As estimações indicam que o efeito crowding-out pode ser observado apenas na crise de 2009, sem ocorrências significativas em 1998 e 2002, conforme especificados nas Tabelas 3 a 5, no Apêndice A. Entretanto, vale uma análise minuciosa no caso brasileiro, pois, sob a mesma especificação de Schult (2002), quanto à transição

$4 \quad$ A variável cor da pele foi considerada apenas para os dados da PME com a nova metodologia: no caso do presente artigo, essa variável foi utilizada apenas na estimação de 2009, pois na metodologia antiga da PME não existe uma variável que caracterize a cor da pele do indivíduo.

5 A atividade industrial foi calculada a partir do índice de produção física industrial, indicador mensurado pela Pesquisa Industrial Mensal Produção Física - Regional (PIM-PF regional) do IBGE. Esse indicador se refere ao total das indústrias de cada estado das respectivas regiões.

6 Essa variável é inserida no modelo porque era necessário considerar uma variável sazonal indicativa de períodos no ano em que usualmente ocorre aumento de contratações temporárias. 
da situação de desocupação para situação de ocupado sob menor qualificação, nota-se que as características relevantes para se sair da situação de desocupação mudaram nas crises de 1998, 2002 e 2009.

Considerando o ano de 1998, as características relevantes do desocupado eram gênero, idade, idade ao quadrado, se havia sido empregado anteriormente e se nunca havia trabalhado antes, sendo que apenas a idade indicava aumento na probabilidade do desocupado encontrar trabalho, ou seja, quanto mais jovem, maior a sua chance de encontrar trabalho. As demais variáveis indicam uma relação de queda na probabilidade do desocupado encontrar trabalho. Nota-se que, em 1998, as características estatisticamente relevantes estavam mais relacionadas às características do trabalhador, e não necessariamente às variáveis como taxa de desocupação e a interativa da variação da atividade industrial de cada região, que representavam condições do mercado de trabalho da região.

Dada observação é relevante, pois, de acordo com Marquetti (2002), o Brasil passou por um processo de desindustrialização nas décadas de 1980 e 1990, tanto em nível de emprego, quanto em valor agregado. Sendo assim, as características significativas em 1998 podem representar não somente aspectos da crise desse ano que tornaram os desocupados mais vulneráveis, mas também características que já mostravam sua vulnerabilidade ante as condições do mercado de trabalho da década de 1990.

Por sua vez, em 2002, as características relevantes se modificaram, uma vez que foram consideradas significativas as variáveis: prêmio, cor da pele, nunca trabalhou antes e RM de Salvador. Assim, os trabalhadores desocupados e que encontraram trabalho na RM de Salvador estavam menos propensos a essa transição quando comparada à RM de São Paulo, indicando a importância da indústria para aquela região. Entre 1996 e 2002, a indústria baiana, não apenas em Salvador, cresceu a taxas superiores à taxa nacional (UDERMAN, 2005), passando a apresentar importantes ramos industriais além do polo petroquímico de Camaçari. No entanto, mesmo diante de um cenário de crescimento do setor industrial, Salvador apresentou, em geral, menores chances de transição da condição de desocupação para emprego.

Em 2002, as demais variáveis significativas apresentaram uma relação direta com a probabilidade de transição da condição de desocupado para ocupado. Nesse caso, chama a atenção a variável prêmio, pois quanto maior a razão entre o salário médio dos trabalhadores qualificados, comparado à média dos menos qualificados, maior a probabilidade de transição da condição do indivíduo desocupado para ocupado. Esse fato retrata o que ocorreu em 2002, segundo dados apresentados a respeito do rendimento médio nominal de acordo com os anos de estudo, conforme Ramos e Valverde (2012): é possível verificar que a variável prêmio demonstrava queda desde 1998, contrapondo uma elevação de 4,66 p.p. 
de 2001 para 2002. Por outro lado, a taxa de desocupação em 2002 foi maior entre trabalhadores qualificados e menor entre trabalhadores menos qualificados. Sendo assim, a taxa de desocupação, mesmo sendo superior, não impactou de forma a reduzir a variável prêmio em 2002.

Tendo em vista o ano de 2009, nota-se uma alteração nas características significativas, mas com um destaque: o efeito crowding-out tornou-se relevante. As variáveis relevantes foram "proporção de desocupados que foram para ocupações de menor qualificação", "gênero", "nunca trabalhou antes" e a RM de Belo Horizonte. Entre elas, os trabalhadores que informam "nunca trabalharam antes" ou trabalhadores que vivem na RM de Belo Horizonte - variável que considera peculiaridades da região e a variação industrial ocorrida nesse ano - apresentam uma probabilidade maior de transição da desocupação para a ocupação. Para as demais variáveis significativas nota-se uma redução na probabilidade de transição da desocupação para a ocupação. As mulheres apresentam uma probabilidade menor de mudança do status de desocupação para ocupação nas crises de 1992, 2002 e 2009. Todavia, observa-se que a variável prêmio não é significante. Nesse contexto, é relevante compreender que a crise de 2009 apresentou peculiaridades distintas das de 1998 e 2002, em especial no tocante ao efeito crowding-out, uma vez que se torna preocupante verificar se esse efeito continuará a ocorrer, pois, mesmo sob uma taxa de desocupação menor em 2009, quando se compara com 1998 e 2002, a variável prêmio se elevou em 37,6\% sob um cenário de queda do nível de desocupação.

Ainda no tocante ao prêmio por qualificação e ao efeito crowding-out, nota-se que no contexto das três recessões não houve uma predominância de ambos simultaneamente. Ou seja, em 1998 ambos não foram estatisticamente relevantes para afetar a probabilidade de transição dos desocupados para o status de ocupado. Em 2002, houve uma redução considerável $(-38,4 \%)$ na proporção de desocupados que foram para ocupações de menor qualificação em relação aos desocupados que foram para ocupações de maior qualificação, enquanto o prêmio reduziu $11,44 \%$. Entretanto, quando se compara 2009 com 2002, o comportamento foi distinto, ou seja, o prêmio subiu em 37,6\% e a "proporção" caiu em 6,2\%, sendo que em 2009 apenas a queda da proporção foi estatisticamente relevante. Isso mostra que os cenários em 1998, 2002 e 2009 são distintos para a análise de ambas as variáveis, mais especificamente em 2002 e 2009. Ou seja, o efeito do aumento do prêmio por qualificação não foi suficiente para "neutralizar" o efeito crowding-out que ocorreu nas RM em 2009. 


\section{Considerações Finais}

O presente trabalho buscou verificar se o efeito crowding-out ocorreu no Brasil nas recessões de 1998, 2002 e 2009. De acordo com os resultados apresentados, esse efeito ocorreu apenas em 2009, se aproximando aos achados de Pecora (2012), que observa como o aumento da oferta de trabalhadores qualificados contribuiu para reduzir o diferencial de salários entre esses trabalhadores e os de menor qualificação, durante os anos 2000. Assim, na escassez de postos de trabalho qualificados, os trabalhadores de melhor qualificação aceitam trabalhar em ocupações de menor qualificação e, consequentemente, de menor remuneração.

No tocante ao prêmio por qualificação, se demonstrou relevante apenas quando houve sua deterioração, em consonância com os resultados apresentados por Fernandes e Menezes (2012). Entretanto, vale lembrar que quando houve aumento do prêmio por qualificação, este não se apresentou estatisticamente relevante.

Considerando os contextos das três recessões, seria adequado verificar melhor a relação entre o prêmio e o efeito crowding-out, pois, quando o prêmio era baixo e o efeito crowding-out era elevado, nenhum se mostrou relevante nos contextos. Contudo, quando houve deterioração e queda no percentual de indivíduos sob o efeito crowding-out, o prêmio mostrou-se relevante. Por fim, quando houve elevação no prêmio, e redução no percentual de indivíduos sob o efeito crowding-out, esse efeito foi significante. Isso indica que a natureza das duas crises de 2002 e 2009 foi distinta e afetou o mercado de trabalho no Brasil diferentemente. Ademais, foi possível observar que, mesmo ocorrendo o efeito crowding-out, este sucedeu de forma a reduzir a probabilidade de transição, ou seja, deixando o cenário ainda mais vulnerável para indivíduos que estavam desocupados.

A expansão acentuada da oferta de trabalhadores qualificados durante os anos 2000 foi preponderante para a constatação do efeito durante a crise de 2009. Logo, o presente estudo corrobora a situação precária dos trabalhadores de menor qualificação no país. Uma política pública que vise capacitar e melhorar a qualificação desses indivíduos é urgente para que tal quadro não piore cada vez mais. Ações como o Programa Nacional de Acesso ao Ensino Técnico e Emprego (Pronatec) do governo federal representam avanços nessa direção, entretanto, não serão suficientes caso também não seja revertida a questão da escassez de postos de trabalho qualificados.

\section{Referências}

AUTOR, D.; LEVY, F.; MURNANE, R. The skill content of recent technological change: an empirical exploration. Quarterly Journal of Economic, v. 118, n. 4, p. 1279-1333, 2003. 
BERMAN, E.; BOUND, J.; GRILICHES, Z. Changes in the demand of skilled labor within U.S. manufacturing: evidence from the annual survey of manufactures. Quarterly Journal of Economics, v. 109, n. 2, p. 367-397, 1994.

BIEWEN, M.; WILKE, R. Unemployment duration and the length of entitlement periods for unemployment benefits: do the job employment subsample and the German socio-economic panel yield the same results? Mannheim: Centre for European Economic Research (ZEW), 2005. (Discussion paper n. 5).

BLOSSFELD, H. P.; ROHWER, G. Techniques of event history modeling: new approaches to causal analysis. Mahwah: Lawrence Erlbaum Associates, 2002.

CARD, D.; LEMIEUX, L. Can falling supply explain the rising return to college for younger? Quarterly Journal of Economics, v. 116, n. 2, p. 705-746, 1996.

DINARDO, J.; FORTIN, N. M.; LEMIEUX, T. Labor market institutions and the distribution of wages, 1973-1992: a semiparametric approach. Econometrica, v. 64, n. 5, p. 1001-1044, 1996.

DOLADO, J.; FELGUEROSO, F.; JIMENO, J. F. Explaining youth labor market problems in Spain: crowding-out, institutions, or technology shifts? Germany: IZA. 2000. (Discussion paper, n. 142).

FERNANDES, R.; MENEZES FILHO, N. Educação, salários e a alocação de trabalhadores entre tarefas: teoria e evidências para o Brasil. Pesquisa e Planejamento Econômico, Rio de Janeiro, v. 42, n. 3, p. 311-328, 2012.

GIOVAETTI, B.; MENEZES FILHO, N. Trade liberalization and demand for skill in Brazil, Economía (LACEA), v. 7, n. 1, p. 1-28, 2006.

IBGE. Pesquisa Industrial Mensal Produção Física - PIM-PF. Diversos anos. Disponível em: $<\mathrm{ftp}$ ://ftp.ibge.gov.br/Industrias_Extrativas_e_de_Transformacao/Pesquisa_Industrial Mensal_Producao_Fisica/Fasciculos/Fasciculo_Indicadores_IBGE_Regional $>$. Acesso em: $\overline{8}$ jul. 2015a.

Pesquisa Mensal de Emprego - PME. Diversos anos. Microdados. Disponível em: <ftp://ftp.ibge.gov.br/Trabalho_e_Rendimento/Pesquisa_Mensal_de_Emprego/ Microdados $>$. Acesso em: 8 jul. 2015b.

KATZ, L.; MARGO, R. Technical change and the relative demand for skilled labor: the united states in historical perspective. Cambridge: National Bureau of Economics, 2013. 69 p. (Working paper n. 18752).

KATZ, L.; MURPHY, K. Changes in relative wages: supply and demand factors. Quarterly Journal of Economics, v. 107, n. 1, p. 35-78, Feb. 1992.

MANACORDA, M.; SÁNCHEZ PÁRAMO, C.; SCHADY, N. Changes in returns to education in Latin America: the role of demand and supply in Latin America, Industrial and Labor Relations Review, Ithaca, v. 63, n. 2, p. 307-326, Jan. 2010.

MARQUETTI, A. Progresso técnico, distribuição e crescimento na economia brasileira: 1955-1998. Estudos Econômicos, v. 32, n. 1, 2002. 
MENEZES FILHO, N.; RODRIGUES, M. Tecnologia e demanda por qualificação na indústria brasileira. Revista Brasileira de Economia, Rio de Janeiro, v. 57, n. 3, p. 569 - 603, jan. 2003.

NICKELL, S. Dynamic models of labour demand. In: ASHENFELTER, O.; LAYARD, R. (Eds.). Handbook of Labor Economics, Amsterdam: North Holland, 1986. p. 473-522. cap. 9. v. 1.

NICKELL, S.; BELL, B. The collapse in demand for the unskilled and unemployed across the OECD. Oxford Review of Economic Policy, v. 11, n. 1, p. 40-62, 1995.

OI, W.Y. Labor as a quasi-fixed factor. Journal of Political Economy, v. 70, n. 6, p. 538-555, 1962.

PECORA, A. R. O papel da oferta e da demanda por qualificação na evolução do diferencial de salários por nível educacional no Brasil. 2012. 68 f. Dissertação (Mestrado em Economia) - Faculdade de Economia, Administração e Contabilidade, Universidade de São Paulo, São Paulo, 2012.

RAMOS, C. A.; VALERDE, R. Sobre o debate da falta de mão-de-obra qualificada no Brasil. Brasília/Salvador: Portal da Indústria, 48 p. 2012. Disponível em: < http://arquivos. portaldaindustria.com.br/app/conteudo_24/2013/08/29/409/20130829160916182383i.pdf>. Acesso em: 8 jul. 2015.

REIS, M. C. Mudanças no desemprego e nos rendimentos do trabalho por nível de qualificação no Brasil. Pesquisa e Planejamento Econômico, v. 36, n. 1, 2006 a.

. Os impactos das mudanças na demanda por trabalho qualificado sobre o desemprego por nível de qualificação durante os anos noventa no Brasil. Revista Brasileira de Economia, Rio de Janeiro, v. 60, n. 3, 2006b.

RIBAS, R. P.; SOARES, S. S. D. Sobre o painel da pesquisa mensal de emprego (PME) do IBGE. Rio de Janeiro: IPEA, 2008. (Texto para discussão n. 1348).

RODRIGUES, E. S.; OLIVEIRA, A. M. H. C.; ALBUQUERQUE, E. M. Uma análise da mobilidade ocupacional no Brasil segundo o nível tecnológico das ocupações. 2008. Trabalho apresentado no $16^{\circ}$ Encontro ABEP, Caxambu, MG, 2008. Disponível em: < http://ww.abep. nepo.unicamp.br/encontro2008/docsPDF/ABEP2008_1225.pdf>. Acesso em: 10 jul. 2014.

SCHULT, P. Crowding-out of unskilled workers in the business cycle: evidence from West Germany. European Sociological Review, v. 21, n. 5, p. 467-480, Dec. 2005.

THUROW, L. C. Generating inequality. Mechanisms of distribution in the US economy. New York: Basic Books, 1975.

UDERMAN, S. A indústria de transformação na Bahia: características gerais e mudanças estruturais recentes. Revista Desenbahia, Salvador, v. 2, n. 3, p. 7-34, 2005. 


\section{Apêndice A}

Tabela 3 - Efeito crowding-out em 1998

\begin{tabular}{|c|c|c|c|c|c|c|c|}
\hline \multicolumn{8}{|c|}{$\begin{array}{l}\text { Saída da situação de desocupação para a situação de empregado sob menor qualificação em } 1998 \\
\text { (especificação Hazard: log normal) }\end{array}$} \\
\hline \multirow[b]{2}{*}{ Taxa de desocupacão } & \multirow{2}{*}{$\begin{array}{c}\text { Razão } \\
\text { Hazard } \\
0,95205\end{array}$} & \multirow{2}{*}{$\begin{array}{c}\begin{array}{c}\text { Erro padrão } \\
\text { robusto }\end{array} \\
0,04215\end{array}$} & \multirow{2}{*}{$\begin{array}{r}\text { Estatística - } \mathrm{z} \\
-1,11\end{array}$} & \multicolumn{2}{|l|}{ Probabilidade } & \multicolumn{2}{|c|}{$\begin{array}{c}\text { Intervalo de } \\
\text { confiança }(95 \%)\end{array}$} \\
\hline & & & & 0,267 & & 0,87292 & 1,03836 \\
\hline Menor qualificação e fazendo curso & 1,20719 & 0,21172 & 1,07 & 0,283 & & 0,85603 & 1,7024 \\
\hline $\begin{array}{l}\text { Interação (nível de qualificação x taxa } \\
\text { de desocupação) }\end{array}$ & 0,99821 & 0,00374 & $-0,48$ & 0,633 & & 0,9909 & 1,00558 \\
\hline $\begin{array}{l}\text { Proporção de desocupados que foram } \\
\text { para ocupaç̃es de menor qualificacão }\end{array}$ & 0,98775 & (2) & $-0,7$ & 0,486 & & (9) & \\
\hline $\begin{array}{l}\text { Prêmio } \\
\text { Pro }\end{array}$ & 1,08458 & 0,14424 & 0,61 & 0,542 & & 0,83571 & 1,40755 \\
\hline Gênero & 1,16691 & 0,07583 & 2,38 & 0,018 & $\begin{array}{l}* * \\
* *\end{array}$ & 1,02736 & 1,32542 \\
\hline Idade & 0,9497 & 0,01738 & $-2,82$ & 0,005 & $*$ & 0,91624 & 0,98438 \\
\hline Idade ao quadrado / 100 & 1,06576 & 0,02692 & 2,52 & 0,012 & $\begin{array}{l}* * \\
* *\end{array}$ & 1,01428 & 1,11985 \\
\hline Empregado anteriormente & 3,44892 & 0,8022 & 5,32 & 0,000 & $*$ & 2,18624 & 5,44087 \\
\hline $\begin{array}{l}\text { Proporção de indivíduos que estavam } \\
\text { em treinamento ou buscando melhor } \\
\text { qualificação }\end{array}$ & 0,93786 & 0,10394 & $-0,58$ & 0,563 & $* *$ & 0,75475 & 1,1654 \\
\hline Nunca trabalhou antes & 1,45854 & 0,20962 & 2,63 & 0,009 & $*$ & 1,10049 & 1,93308 \\
\hline Recife & 0,98959 & 0,00708 & $-1,46$ & 0,144 & & 0,97581 & 1,00357 \\
\hline Salvador & 0,98289 & 0,01191 & $-1,42$ & 0,154 & & 0,95983 & 1,00651 \\
\hline Belo Horizonte & 1,00484 & 0,0055 & 0,88 & 0,377 & & 0,99412 & 1,01568 \\
\hline Rio de Janeiro & 0,99818 & 0,00302 & $-0,6$ & 0,547 & & 0,99227 & 1,00412 \\
\hline Porto Alegre & 0,99772 & 0,00367 & $-0,62$ & 0,534 & & 0,99056 & 1,00493 \\
\hline Dezembro & 0,98167 & 0,08891 & $-0,2$ & 0,838 & & 0,822 & 1,17236 \\
\hline Número de observações na & & & & & & & 1705 \\
\hline Número d & ntinuars & lesocupadas & & & & & 901 \\
\hline Teste Wald (12) & & & & & & & 62,13 \\
\hline Prob $>$ chi2 $=$ & & & & & & & 0,0000 \\
\hline
\end{tabular}

Fonte: Elaboração própria a partir dos microdados da PME (IBGE, 2015b). 
Tabela 4 - Efeito crowding-out em 2002

\begin{tabular}{|c|c|c|c|c|c|c|c|}
\hline \multicolumn{8}{|c|}{$\begin{array}{l}\text { Saída da situação de desocupação para a situação de empregado sob menor qualificação em } 2002 \\
\text { (especificação Hazard: log normal) }\end{array}$} \\
\hline & $\begin{array}{l}\text { Razão } \\
\text { Hazard }\end{array}$ & $\begin{array}{l}\text { Erro } \\
\text { padrão } \\
\text { robusto }\end{array}$ & $\begin{array}{l}\text { Estatística } \\
\quad \mathrm{z}\end{array}$ & \multicolumn{2}{|l|}{ Probabilidade } & \multicolumn{2}{|c|}{$\begin{array}{c}\text { Intervalo de } \\
\text { confiança }(95 \%)\end{array}$} \\
\hline Taxa de desocupação & 1,1519 & 0,0724 & 2,2500 & 0,0240 & & 1,0185 & 1,3028 \\
\hline Menor qualificação e fazendo curso & 0,8318 & 0,3492 & $-0,4400$ & 0,6610 & & 0,3654 & 1,8939 \\
\hline $\begin{array}{l}\text { Interação (nível de qualificação x taxa de } \\
\text { desocupação) }\end{array}$ & 0,9967 & 0,0125 & $-0,2600$ & 0,7940 & & 0,9726 & 1,0215 \\
\hline $\begin{array}{l}\text { Proporção de desocupados que foram para } \\
\text { ocupaços de menor qualificação }\end{array}$ & 0,9937 & 0,0096 & $-0,6600$ & 0,5090 & & 0,9751 & 1,0126 \\
\hline Prêmio & 0,9240 & 0,0433 & $-1,6900$ & 0,0910 & * & 0,8430 & 1,0128 \\
\hline Gênero & 1,1026 & 0,1247 & 0,8600 & 0,3880 & & 0,8834 & 1,3762 \\
\hline Cor de pele & 0,7652 & 0,0928 & $-2,2100$ & 0,0270 & ** & 0,6033 & 0,9705 \\
\hline Idade & 0,9601 & 0,0465 & $-0,8400$ & 0,4010 & & 0,8732 & 1,0558 \\
\hline Idade ao quadrado / 100 & 1,0568 & 0,0754 & 0,7700 & 0,4390 & & 0,9189 & 1,2154 \\
\hline Empregado anteriormente & 2,2037 & 1,2688 & 1,3700 & 0,1700 & & 0,7130 & 6,8112 \\
\hline $\begin{array}{l}\text { Proporção de indivíduos que estavam em } \\
\text { treinamento ou buscando melhor }\end{array}$ & & & & & & & \\
\hline qualificação & 1,2537 & 0,4710 & 0,6000 & 0,5470 & & 0,6004 & 2,6179 \\
\hline Nunca trabalhou antes & 0,3848 & 0,0524 & $-7,0100$ & 0,0000 & $* * *$ & 0,2946 & 0,5027 \\
\hline Recife & 1,0793 & 0,0657 & 1,2500 & 0,2100 & & 0,9579 & 1,2162 \\
\hline Salvador & 1,0181 & 0,0073 & 2,4900 & 0,0130 & $* *$ & 1,0038 & 1,0327 \\
\hline Belo Horizonte & 0,9919 & 0,0106 & $-0,7600$ & 0,4480 & & 0,9713 & 1,0130 \\
\hline Rio de Janeiro & 0,9983 & 0,0025 & $-0,6700$ & 0,5030 & & 0,9934 & 1,0033 \\
\hline Porto Alegre & 1,0019 & 0,0061 & 0,3200 & 0,7490 & & 0,9901 & 1,0140 \\
\hline Dezembro & 0,8388 & 0,1505 & $-0,9800$ & 0,3270 & & 0,5901 & 1,1924 \\
\hline Número de observações na amostra & & & & & & & 422 \\
\hline Número de observações na amostra que cor & uaram de & socupadas & & & & & 279 \\
\hline Teste Wald (12) & & & & & & & 96,57 \\
\hline Prob $>$ chi2 $=$ & & & & & & & 0,0000 \\
\hline
\end{tabular}

Fonte: Elaboração própria a partir dos microdados da PME (IBGE, 2015b). 
Tabela 5 - Efeito crowding-out em 2009

\begin{tabular}{|c|c|c|c|c|c|c|c|}
\hline \multicolumn{8}{|c|}{ Saída da situação de desocupação para a situação de empregado sob menor qualificação em 2009 (especificação Hazard: log normal) } \\
\hline & $\begin{array}{l}\text { Razão } \\
\text { Hazard }\end{array}$ & $\begin{array}{l}\text { Erro } \\
\text { padrão } \\
\text { robusto }\end{array}$ & Estatística - z & Probabilidade & \multicolumn{3}{|c|}{$\begin{array}{l}\text { Intervalo de } \\
\text { confiança } \\
(95 \%)\end{array}$} \\
\hline Taxa de desocupação & 0,9688 & 0,0561 & $-0,5500$ & 0,5840 & & 0,8650 & 1,0852 \\
\hline Menor qualificação e fazendo curso & 1,3155 & 0,5136 & 0,7000 & 0,4830 & & 0,6119 & 2,8278 \\
\hline Interaçãoo (nivel de qualificação x taxa de desocupação) & 0,9918 & 0,0098 & $-0,8300$ & 0,4040 & & 0,9727 & 1,0112 \\
\hline Proporção de desocupados que foram para ocupações de menor qualificação & 1,0945 & 0,0506 & 1,9500 & 0,0510 & * & 0,9996 & 1,1984 \\
\hline Prêmio & 0,9078 & 0,1227 & $-0,7200$ & 0,4740 & & 0,6964 & 1,1833 \\
\hline Gênero & 1,2824 & 0,1332 & 2,3900 & 0,0170 & ** & 1,0461 & 1,5720 \\
\hline Cor de pele & 1,0001 & 0,1096 & 0,0000 & 0,9990 & & 0,8069 & 1,2397 \\
\hline Idade & 0,9828 & 0,0328 & $-0,5200$ & 0,6030 & & 0,9206 & 1,0492 \\
\hline Idade ao quadrado / 100 & 1,0193 & 0,0474 & 0,4100 & 0,6810 & & 0,9305 & 1,1166 \\
\hline Empregado anteriormente & 1,5694 & 0,6244 & 1,1300 & 0,2570 & & 0,7196 & 3,4227 \\
\hline $\begin{array}{l}\text { Proporção de indivíduos que estavam em treinamento ou buscando melhor } \\
\text { gualificacão }\end{array}$ & & & & & & & \\
\hline $\begin{array}{l}\text { qualificação } \\
\text { Nunca trabalhou antes }\end{array}$ & $\begin{array}{l}0,9620 \\
0,3827\end{array}$ & $\begin{array}{l}0,3009 \\
0,0533\end{array}$ & $\begin{array}{l}-0,1200 \\
-6,8900\end{array}$ & $\begin{array}{l}0,9010 \\
0,0000\end{array}$ & *** & $\begin{array}{l}0,5211 \\
0,2912\end{array}$ & $\begin{array}{l}1,7759 \\
0,5028\end{array}$ \\
\hline Recife & 0,9837 & 0,0539 & $-0,3000$ & 0,7640 & & 0,8835 & 1,0952 \\
\hline Salvador & 1,0021 & 0,0076 & 0,2800 & 0,7770 & & 0,9874 & 1,0171 \\
\hline Belo Horizonte & 0,9865 & 0,0081 & $-1,6700$ & 0,0950 & * & 0,9708 & 1,0024 \\
\hline Rio de Janeiro & 0,9969 & 0,0031 & $-1,0000$ & 0,3190 & & 0,9910 & 1,0030 \\
\hline Porto Alegre & 0,9914 & 0,0055 & $-1,5600$ & 0,1180 & & 0,9808 & 1,0022 \\
\hline Dezembro & 0,8450 & 0,1347 & $-1,0600$ & 0,2910 & & 0,6183 & 1,1548 \\
\hline Número de observações na amostra & & & & & & & 641 \\
\hline Número de observações na amostra que continuaram desocupadas & & & & & & & 350 \\
\hline Teste Wald (12) & & & & & & & 75,79 \\
\hline Prob $>$ chi2 $=$ & & & & & & & 0,0000 \\
\hline
\end{tabular}

Fonte: Elaboração própria a partir dos microdados da PME (IBGE, 2015b).

Recebido em: 05/05/2014.

Aceito em: 11/09/2014. 